L'HOMME L'Homme

187-188 | 2008

Miroirs transatlantiques

\title{
Malentendus transatlantiques
}

La tradition de Chicago, Park et la sociologie française

Jean-Michel Chapoulie

\section{CpenEdition}

\section{Journals}

Édition électronique

URL : https://journals.openedition.org/lhomme/29267

DOI : 10.4000//homme.29267

ISSN : 1953-8103

Éditeur

Éditions de l'EHESS

\section{Édition imprimée}

Date de publication : 3 octobre 2008

Pagination : 223-246

ISBN : 978-2-7132-2186-6

ISSN : 0439-4216

Référence électronique

Jean-Michel Chapoulie, "Malentendus transatlantiques », L'Homme [En ligne], 187-188 | 2008, mis en ligne le 01 janvier 2010, consulté le 23 avril 2022. URL : http://journals.openedition.org/lhomme/ 29267 ; DOI : https://doi.org/10.4000//homme.29267 
chercher : repérer : avancer

Cet article est disponible en ligne à l'adresse :

http://www.cairn.info/article.php?ID REVUE=LHOM\&ID NUMPUBLIE=LHOM 187\&ID ARTICLE=LHOM 1870223

Malentendus transatlantiques. La tradition de Chicago, Park et la sociologie française

par Jean-Michel CHAPOULIE

| Éditions de l'EHESS | L'Homme

$2008 / 3-4-n^{\circ} 187-188$

ISSN 0439-4216 | ISBN 9782713221866 | pages 223 à 246

Pour citer cet article :

-Chapoulie J.-M., Malentendus transatlantiques. La tradition de Chicago, Park et la sociologie française, L'Homme 2008/3-4, n० 187-188, p. 223-246.

Distribution électronique Cairn pour les Éditions de l'EHESS.

(C) Éditions de l'EHESS. Tous droits réservés pour tous pays.

La reproduction ou représentation de cet article, notamment par photocopie, n'est autorisée que dans les limites des conditions générales d'utilisation du site ou, le cas échéant, des conditions générales de la licence souscrite par votre établissement. Toute autre reproduction ou représentation, en tout ou partie, sous quelque forme et de quelque manière que ce soit, est interdite sauf accord préalable et écrit de l'éditeur, en dehors des cas prévus par la législation en vigueur en France. Il est précisé que son stockage dans une base de données est également interdit. 


\section{Malentendus transatlantiques \\ La tradition de Chicago, Park et la sociologie française}

Jean-Michel Chapoulie

M

ÊME SI LES SCIENCES SOCIALES ont cherché à adopter dès le début du siècle le modèle d'organisation des sciences de la nature, avec ses contacts entre chercheurs de différentes nationalités, la communication transfrontalière entre chercheurs en sciences sociales a été et reste difficile. Pour ces disciplines, les questions d'actualité, les tendances d'évolution, les méthodes documentaires en vogue à un moment donné, les modes de rédaction des analyses ne sont pas souvent les mêmes des deux côtés de celles-ci. Ce fait est sans doute spécialement frappant pour la sociologie, plus dépendante des débats publics d'une société à laquelle appartiennent par ailleurs les chercheurs.

On ne saurait donc s'étonner de la lenteur et des incertitudes de la diffusion qui caractérisent, en sociologie, même les œuvres traitées comme classiques - c'est-à-dire répétitivement inscrites dans les programmes d'enseignement et les histoires conventionnelles de la sociologie. Les incompréhensions et les contresens qui accompagnent si souvent le passage des frontières des travaux sociologiques ont cependant leurs contreparties : ils offrent une voie d'accès à la compréhension de ce qui reste implicite dans les analyses de sciences sociales ${ }^{1}$. Les considérant comme une ressource et non comme un obstacle, Marc Bloch (1992 [1949] : 64) mettait en avant les expériences individuelles des chercheurs en invoquant un "emprunt" aux "expériences quotidiennes » comme un élément du travail d'historien. Je me propose ici de dégager ce qui, dans ces expériences individuelles, renvoie à ces éléments collectifs que sont les questionnements et représentations sociales constitués. Pour mettre en évidence ces éléments collectifs, je développerai l'exemple de la réception des analyses de la «tradition de Chicago " par la sociologie française, et plus précisément de la quasi non-réception des

1. Une analyse de certains de ces éléments, comme le rapport des chercheurs à la culture savante, se trouve dans Bourdieu (1997). 
éléments centraux de celle-ci que représentent les analyses des « relations de races et de culture » dans le cadre analytique proposé par Robert Park².

Les contacts des sociologues français avec les sociologues de la tradition de Chicago peuvent être reconstitués à partir des comptes rendus de voyage et mémoires, des traductions, notes critiques, et références. Je m’appuie ici essentiellement sur le dépouillement d'ouvrages et des principales revues de sociologie: pour la période antérieure à 1940, les Annales sociologiques, les Cahiers internationaux de sociologie (1945-1965), la Revue française de sociologie (1960-1980) ${ }^{3}$.

Parmi les éléments susceptibles de retenir l'attention des chercheurs lors de ces contacts, on peut distinguer entre schèmes généraux d'analyse - notions, hypothèses sur les relations entre celles-ci, interrogations - et méthodes de recueil et de traitement de la documentation. Dans une seconde étape de cette recherche, j'ai examiné les publications françaises sur l'immigration, le domaine de recherche homologue pour la sociologie française de celui des relations de races et de culture pour la sociologie américaine. J'ai procédé pour cela au dépouillement de la revue Population et de la collection des «Cahiers de l'INED ».

Je présenterai d'abord rapidement les recherches de la tradition de Chicago en sociologie, ainsi que quelques repères sur l'histoire de la sociologie américaine et la place qu'y occupent les recherches sur les relations de races et de culture ${ }^{4}$.

\section{Park, la tradition de Chicago et l'évolution de la sociologie américaine}

C'est à l'Université de Chicago qu'est née, à la fin du XIX siècle, la première entreprise de recherches empiriques sur la société contemporaine installée dans une université américaine. Les débuts furent difficiles, et c'est seulement en 1918 que parait un premier ouvrage significatif : la monographie de William I. Thomas et Florian Znaniecki sur l'émigration polonaise aux États-Unis ${ }^{5}$. Les recherches

2. L'expression "relations de race et de culture" n'est pas utilisée par Park, mais par les chercheurs de la génération suivante. Il faut peut-être préciser pour un lecteur francophone contemporain que le terme « race » n'a aucune connotation biologique dans son usage le plus fréquent par les sciences sociales américaines après 1930. La définition donnée par un élève de Park peut servir de référence : «La race désigne simplement un groupe de personnes qui sont considérées et traitées dans la vie courante comme une race. L'appartenance à la race correspond simplement aux individus qui sont identifiés et classés comme lui appartenant» (Blumer 1955: 4). J'ai utilisé parfois le terme «relations ethniques » à la place du terme "relations de race » en laissant de côté des distinctions que l'on trouve dans certaines recherches américaines des cinquante dernières années.

3. Au cours de la période 1945-1970, la sociologie n'est pas clairement séparée de l'anthropologie et les trois revues - surtout les Cahiers internationaux de sociologie - publient des articles d'anthropologues. La première revue française généraliste d'anthropologie, L'Homme, n'est créée, rappelons-le, qu'en 1961.

4. Une analyse historique détaillée de la tradition de Chicago dans son contexte se trouve dans Chapoulie (2001). La démarche historique, et non hagiographique ou de critique présentiste, de cet ouvrage est présentée dans Chapoulie (2005).

5. The Polish Peasant in Europe and America, New York, A. Knopf, 1927 [1918-1920], 5 vol. Deux traductions partielles existent en français : la traduction du tome 3: Le Paysan polonais en Europe et en Amérique. Récit de vie d'un migrant, Paris, Nathan, 1998, et un choix de textes: Fondation de la sociologie américaine. Morceaux choisis, Paris, L’Harmattan, 2000. 
empiriques sur la société américaine contemporaine connaissent un développement rapide après 1918, sous l'inspiration de cet ouvrage et de l'enseignement de Robert Park (1864-1944), un ancien journaliste qui, en collaboration avec un jeune collègue, Ernest Burgess, publie un manuel en son temps qualifié de «Bible verte " (Park \& Burgess 1921). L'ouvrage propose un cadre de notions pour effectuer des recherches empiriques sur la société américaine contemporaine. Les mêmes auteurs publient un autre volume (Park \& Burgess 1925) destiné à promouvoir des études sur les villes, peu après que l'une des fondations Rockefeller eut proposé les premiers financements importants pour des recherches en sciences sociales. Ce financement permit, entre autres, la publication d'une collection d'ouvrages, issus le plus souvent de thèses $(\mathrm{PhD})$ en sociologie soutenues à l'Université de Chicago inspirées par Park. Ces ouvrages portent sur des sujets comme les bandes de jeunes de Chicago, un quartier de la ville juxtaposant zone résidentielle habitée par l'élite et zone d'habitats d'immigrants pauvres, un type particulier de dancing, ou encore l'étiquette des relations entre les races dans le Sud, le mélange de populations dans les îles Hawaï, etc. Ces monographies s'appuient souvent en partie sur un contact direct des chercheurs avec les phénomènes étudiés, une démarche nouvelle pour la sociologie américaine jusque-là portée aux compilations de bibliothèque. Cette démarche est prônée par Robert Park, qui l'emprunte au journalisme d'enquête, mais elle sera un peu plus tard identifiée à la démarche ethnographique ${ }^{6}$.

Les sociologues de Chicago recueillent fréquemment des autobiographies (life history) - deux ouvrages sont par exemple consacrés à la présentation et au commentaire d'autobiographies de jeunes délinquants. Celles-ci, comme les interviews et les observations qui permettent de réaliser des case studies, sont destinées à appréhender les expériences subjectives qui sont la voie d'accès à ce que Park désigne par le terme "ordre moral». Les monographies utilisent aussi une documentation variée collectée par différentes administrations et associations à but social, à la presse, etc. Elles exploitent parfois des statistiques et surtout des représentations cartographiques de la localisation des phénomènes étudiés dans les villes - principalement Chicago. Dans la terminologie introduite par Park, il s'agit par là d'étudier "l'ordre écologique », celui que constituent sur le terrain les relations de concurrence entre groupes sociaux pour l'utilisation de l'espace.

Ces monographies, dont la publication s'étend de 1924 à 1940, représentèrent pour la sociologie américaine le modèle de la recherche empirique. C’est à leurs auteurs, et à ceux qui les ont inspirés (Thomas, Park, Burgess), qu'a été conféré ultérieurement le label "école de Chicago ». On peut regrouper en trois catégories les objets étudiés: les villes, leur développement, les comportements

6. Il n'y a qu'une faible distinction institutionnelle entre anthropologie et sociologie à l'Université de Chicago jusqu'en 1929, et, jusqu'à son éviction, en 1918, William Thomas, dans un département d'anthropologie et de sociologie se place toujours du côté des anthropologues. Dès sa parutioin en 1922, l'ouvrage de Malinowski, Argonauts of the Western Pacific, a été remarqué et apprécié des sociologues de Chicago. 
urbains, avec notamment la "désorganisation sociale " (délinquance, divorce, suicide, etc.) ; les relations de races et de culture; enfin, la presse. Ce dernier sujet qui correspond à un intérêt personnel de Robert Park n'occupe à peu près aucune place dans la postérité de l'école de Chicago en sociologie et je le laisserai de côté.

Park, qui avait 50 ans lors de son recrutement comme sociologue, n'a pas luimême publié de compte rendu de recherche qui aurait pu faire date en sociologie, mais de nombreux essais, ultérieurement réunis en trois volumes (Park 1950, 1952, 1955). Si la ville est un sujet d'intérêt, voire de fascination pour Park, il n’a jamais pour autant mené lui-même de recherches systématiques dans ce domaine. Il n'en va pas de même pour l'étude des relations de race et de culture. Les essais de Park sur ce sujet s'appuient non seulement sur des recherches originales, mais aussi sur une expérience personnelle exceptionnellement riche. Il est l'un des seuls universitaires de sa génération à connaitre ethnographiquement (selon les termes de sa fille, l'anthropologue Margaret Redfield) le monde des Noirs ruraux du Sud - il a été, vers 1905, secrétaire du principal leader noir de la période, Booker Washington. Il a suivi, comme le montrent ses essais, les avatars d'une évolution extrêmement rapide qui correspond au passage d'une population rurale localisée dans les États du Sud en 1900, à une population en partie urbaine établie dans le Nord en 1940. Il s'est également particulièrement intéressé à un autre groupe victime de discriminations aux ÉtatsUnis : les Asiatiques de la Côte Ouest. Parmi les autres exemples retenus de Park, on trouve celui de l'Europe centrale d'avant 1914 analysé par des sociologues bien connus de Park comme Gumplowicz, les Noirs du Brésil (étudié par un de ses élèves), les Îles Hawaï (étudié par un autre).

On sait que la présence sur le même territoire de populations d'origines géographiques diverses, culturellement et religieusement différentes, et dont les places dans la société américaine sont extrêmement variées, constitue depuis 1850 un problème public majeur aux États-Unis, notamment aux yeux du groupe anglosaxon protestant qui occupe une position hégémonique. La perspective proposée par Robert Park sur les relations de races rompt radicalement avec celle qui inspirait les analyses antérieures : contrairement à ses prédécesseurs, l'objet central de Robert Park est le système des relations entre ces groupes et non pas des caractéristiques supposées substantielles de chacun d'entre eux ${ }^{7}$. Les relations de races sont considérées par Park non comme des éléments durablement stables, mais comme soumises à processus d'évolution. Loin de s'enfermer dans le cas particulier des États-Unis, il a développé une perspective comparative large, insistant sur le fait que les contacts entre groupes de race et de culture sont des phénomènes omniprésents dans l'histoire du monde depuis l'Antiquité (Park 1950 [1939]). Comme je l'ai montré ailleurs (Chapoulie 2008), le schéma d'analyse de Park est une sorte d'abstraction de la situation des États-Unis d'avant 1914, avec ses vagues successives d'immigration massive et une minorité hégémonique.

7. Voir Chapoulie (1999) pour une présentation du schéma d'analyse de Park. 
Tous les sociologues qui ont enseigné longtemps ou qui ont été formés à l'Université de Chicago ne se rattachent pas à ce qui a été appelé «la tradition de Chicago ». En 1927, l'université a recruté l'un des spécialistes en vue de l'usage de la démarche statistique en sociologie, William Ogburn, dont la notoriété repose sur ses analyses des grandes évolutions sociales au moyen de séries statistiques. Le milieu des années 1930 marque la fin d'une sorte de domination de l'Université de Chicago sur la sociologie américaine, et le déclin d'un certain type de recherche qu'illustraient les monographies de l'école de Chicago. Une nouvelle source documentaire occupe une place croissante dans la sociologie américaine : les enquêtes par questionnaires conduisant à des exploitations statistiques. Cette démarche, qui est développée après 1940 par Paul Lazarsfeld à l'Université Columbia, passe pour la démarche d'avenir de la sociologie. L'usage d'une documentation variée et le contact direct avec les phénomènes - la démarche privilégiée par Robert Park - connaissent corrélativement un certain discrédit parmi les sociologues car suspectés de manquer de rigueur scientifique. Un nouveau centre d'excellence de la sociologie apparaît aussi après la guerre, à Harvard, autour de Talcott Parsons. Le département de sociologie de l'Université de Chicago reste cependant, après 1945, l'un des principaux producteurs de $\mathrm{PhD}$ en sociologie et un centre actif de recherche. Deux de ceux qui se considèrent comme les élèves de Park, Louis Wirth et Herbert Blumer, y enseignent jusqu'en 1950, et un troisième, Everett Hughes, jusqu'en 1961. Les deux derniers nommés apparaissent rétrospectivement comme des enseignants influents qui forment une troisième génération de sociologues - Howard Becker, Anselm Strauss, Eliot Freidson, Donald Roy, Joseph Gusfield, Erving Goffman, pour citer ceux dont des ouvrages ont été traduits en français. Leur approche, qui s'appuie sur une démarche ethnographique, est différente de celle des recherches des années 1930, mais une certaine continuité avec celle-ci est cependant évidente. J'utilise, après d'autres, l'expression «tradition de Chicago " pour désigner l'ensemble diversifié de ces recherches « autour » de l'Université de Chicago.

Trois des élèves de Park ayant accédé à une notoriété nationale, Franklin Frazier, Everett Hughes et Herbert Blumer ont publié des analyses sur les relations de races. Franklin Frazier, le premier sociologue afro-américain à être élu à la présidence de l'American Sociological Association, a insisté sur sa dette à l'égard de la sociologie de Park - y compris dans son allocution prononcée en 1949 comme président de cette association (Frazier 1949). Quant à Everett Hughes, il a consacré une monographie à une ville industrielle du Canada proche de la frontière linguistique, centrée sur les contacts entre anglophones et francophones. Après 1937, il étudie - le sujet était alors nouveau -, l'introduction de travailleurs noirs dans la grande industrie. Et l'essai qui conclut sa présidence de l'American Sociological Association, en août 1963, à un moment critique de la campagne pour les droits civiques, reprend le schéma d'analyse de Park (Hughes 1963). On voit que les travaux sur les relations de race qui reposent sur le cadre analytique proposé par Robert Park ont fait durablement partie de l'actualité de la sociologie américaine. 
Examiner comment la sociologie française a accueilli ces recherches constitue donc un bon exemple pour mettre en évidence les conditions qui rendent possibles ou impossibles les emprunts à une autre tradition nationale. Je partirai du premier contact attesté, en 1930, entre la sociologie française et la tradition de Chicago.

\section{Maurice Halbwachs et la tradition de Chicago}

Maurice Halbwachs est, après 1918, le seul des élèves de Durkheim à s'intéresser activement à la sociologie en tant que discipline associée à des enquêtes sur le monde contemporain. Avec cet autre durkheimien qu'est François Simiand, il est un fervent partisan de l'usage des statistiques en sociologie. Ses principaux centres d'intérêts portent à la fois sur la démographie, les villes - sa thèse de droit a pour sujet Les Expropriations et le prix des terrains à Paris (1860-1909) - et sur la classe ouvrière - sa thèse d'État ès lettres est consacrée à La Classe ouvrière et les niveaux de vie. À la sociologie de Durkheim, Halbwachs ajoute une attention aux différences de classe et on peut rappeler sa collaboration au journal socialiste $L^{\prime} H u$ manité avant 1914. Professeur de sociologie à l'Université de Strasbourg, il a fait un séjour de trois mois à l'Université de Chicago en 1930, sur l'invitation de William Ogburn. À Chicago, Halbwachs a rencontré Robert Park et Ernest Burgess.

Un article sur la ville de Chicago publié à son retour (Halbwachs 1932) présente le développement de la ville et quelques-unes des caractéristiques pour lesquelles les recensements fournissent des statistiques - habitat, transport. Il décrit les propriétés spécifiques de différents quartiers en s’appuyant sur les monographies des élèves de Park et Burgess, notamment leurs analyses écologiques. C'est la question de la composition ethnique de la ville qui a surtout retenu l'attention d'Halbwachs. Il faut rappeler que les ressources qu'offraient la sociologie de Durkheim pour étudier le contact de populations qui se considèraient comme différentes étaient limitées : la seule mention de la question se trouve dans une discussion, en 1905, où Durkheim propose une distinction entre État (groupement politique englobant) et nationalité (communauté de civilisation) en remarquant que pour la France les deux se confondent (Durkheim 1975: 178-186). Dans la dernière partie de son article, titrée "Chicago, ville d'immigrants", ainsi que dans la conclusion, Halbwachs s'interroge sur «l'assimilation» (il ne définit pas le terme) de ces différentes populations. Il s'appuie sur une comparaison avec Paris, examine "cette notion de race qui n'offre en somme rien d'irréductible ", pour conclure que "plus que par la religion et la langue, les immigrants se distinguent des Américains, et ils se distinguent entre eux, par leur situation ou leur niveau social». Ainsi, selon lui, l'hétérogénéité ethnique n'est qu’un phénomène superficiel qui dissimule les différences de classe :

«Ce n'est par parce qu'étrangers, mais parce que ouvriers, surtout parce que manœuvres et ouvriers de la grande industrie, que la masse des immigrants, admise à résider, est cependant séparée de la vie urbaine, exclue du courant traditionnel et continu qui n'entraîne que les éléments vraiment "bourgeois", ou en relation et en contact intime et familier avec la bourgeoisie" (Halbwachs 1932: 47). 
On comprend pourquoi Halbwachs a laissé de côté les monographies qui portent sur les relations de races, ainsi que les analyses de Park sur le même sujet.

Une documentation exceptionnellement riche permet de connaître plus précisément les réactions d'Halbwachs à ce qu'il a vu de la ville et des recherches des sociologues de Chicago. Christian Topalov a publié dans Genèses la série d'articles donnés presque anonymement par Maurice Halbwachs au Progrès de Lyon (Topalov 2005). On dispose également de la correspondance d'Halbwachs avec sa famille pendant son voyage. C'est sur ce matériel que s'appuie Topalov dans deux articles dont je reprends ici une partie des conclusions (Topalov 2006a, 2006b).

Plusieurs éléments confirment que Maurice Halbwachs a cherché à rabattre la question des différences ethniques sur une dimension sociale qui lui était au contraire familière, l'appartenance de classe, comme elle l'était aux intellectuels français de sa génération et de son orientation politique. L'idée que les différences de classes sont l'élément essentiel de la structure sociale - celui qui détermine l'organisation de la société -, que les divisions ethniques doivent en fin de compte se ramener à celles-ci, apparaît comme l'une des caractéristiques principales de la sociologie de Halbwachs que celui-ci partage avec une grande partie des spécialistes de sciences sociales de son temps, aussi bien en France qu'en Grande-Bretagne ou encore en Allemagne. Une autre possibilité d'interprétation est ignorée par Halbwachs : le principe de structuration ethnique peut composer durablement ses effets avec les différences de classe, sans être réductible à celui-ci. C'est ce que suggérait, selon Hughes (1943), la situation d'une ville de la frontière linguistique du Québec, avec une classe supérieure francophone à côté d'une classe supérieure anglophone distincte de celle-ci par sa place dans la division du travail. On peut aussi relever que les différences de sexe sont ignorées par Halbwachs, tout autant que par les sociologues de Chicago de 1930 ou de 1960 : plus encore que les différences ethniques, il aurait été difficile de les réduire à des différences de classe.

Christian Topalov (2006a : 569) montre que les réactions d'Halbwachs reposent sur «l'élaboration savante d'opinions communes qui trouvent leurs sources dans des situations sociales que le savant partage avec d'autres ", c'est-à-dire que ses observations s'appuient sur des stéréotypes et des schèmes de raisonnements partagés avec la plupart des personnes de sa génération et de son milieu. Topalov conclut que les schèmes élémentaires de perception et d'interprétation du monde social de Halbwachs ont fait écran à sa compréhension des travaux des sociologues de Chicago.

Relevons que Maurice Halbwachs s'est peu intéressé aux méthodes de documentation utilisées par les sociologues de Chicago. Il reproduit dans son article de 1932 la carte de la distribution de la ville selon des zones concentriques établie par Burgess. Halbwachs a ignoré les case studies, et plus encore l'observation et le contact direct. Il a trouvé «idiote» la proposition de Burgess d'aller dans un endroit où l'on pouvait rencontrer des assassins, et il s'est contenté de voir de l'extérieur la ville de Chicago, les rues, les marchés, à la manière d'un touriste.

Le dépouillement des Annales sociologiques - qui prend la suite de L'Année sociologique de Durkheim et dont Halbwachs est le secrétaire - montre que l'intérêt de celui-ci pour la tradition de Chicago ne fut pas durable. Peu d'ouvrages 
qui s'y rattachent sont signalés et aucun ne bénéficie d'une note critique. À peu près aucun ouvrage américain consacré aux Noirs n'est mentionné, et la rubrique bibliographique "Déplacements et migrations de population ", parfois tenue par Halbwachs, est très peu fournie. Seule la monographie de Ruth Cavan (1928) sur le suicide fait l'objet d'un compte rendu d'Halbwachs ${ }^{8}$. Ce dernier n'indique pas qu'elle s'insère dans un ensemble d'autres travaux et son appréciation est plutôt négative.

\section{Les jeunes sociologues français après 1945 et la tradition de Chicago}

À peu près réduite à l'existence de trois ou quatre postes de professeurs de faculté et au centre de documentation sociale de l'École normale supérieure avant 1940, la sociologie connaît un nouveau développement en France, après 1945, avec le recrutement au CNRS d'une nouvelle génération de chercheurs (Chapoulie 1991). Ce sont pour une notable part des élèves ou protégés de Georges Friedmann, professeur au CNAM en 1945, de Georges Gurvitch, professeur de sociologie à Strasbourg, et de Gabriel Le Bras, professeur de droit canonique (pour les sociologues de la religion), et un peu plus tard de Jean Stoetzel (un agrégé de philosophie en contact avec les sociologues de Columbia, qui a introduit en France les sondages d'opinion) et de Raymond Aron. Intellectuellement, les jeunes sociologues sont détachés de l'héritage durkheimien dans le contexte idéologique de reconstruction nationale de la Libération. Gurvitch, qui a passé la période de la guerre aux États-Unis, entretient des contacts avec la sociologie américaine et publie en 1947, en collaboration avec Wilbert E. Moore, une version française d'un ouvrage paru deux ans plus tôt en anglais. Le titre La Sociologie du XXe siècle, indique le contenu : il s'agit d'une présentation de la sociologie telle que la définissent alors les départements de sociologie des universités américaines. Parmi les contributions figurent celles de deux sociologues que l'on peut rattacher à la tradition de Chicago : Ernest Burgess, sur les méthodes de la sociologie; Florian Znaniecki sur les "Organisations sociales et institutions". Les autres articles viennent de représentants de courants très variés de la sociologie américaine (Robert MacIver, Howard P. Becker, Talcott Parsons, Robert K. Merton, etc.). La place accordée aux recherches menées à l'Université de Chicago est réduite, ce qui correspond aux orientations de Gurvitch, peu sensible à la dimension empirique de la discipline, et à celle de W. E. Moore, un sociologue passé par Harvard qui se rattache au courant «fonctionnaliste ».

Une partie des jeunes sociologues recrutés au CNRS après 1947 - notamment Henri Mendras, Alain Touraine, François Bourricaud, Michel Crozier, Éric de Dampierre - ont fréquenté avant 1960 les universités américaines, d'abord les Universités Harvard et Columbia, et en deuxième rang, l'Université du Michigan (où se trouvent des psychologues sociaux alors au sommet de leur notoriété), et

8. Annales sociologiques, série C, fascicule 1, 1935 : 181-182. 
l'Université de Chicago. Chacun de ces sociologues a utilisé à sa manière, en fonction de ses propres sujets de recherche, expériences antérieures, ambitions de carrière, ce qu'il a vu ou appris aux États-Unis. Aucun ne s'est intéressé durablement aux recherches qui s'inscrivent dans la filiation de Park, alors représentée à l'Université de Chicago par Burgess, Wirth, Blumer et Hughes. La réaction d'Henri Mendras, l'un des jeunes chercheurs qui passa une année à l'Université de Chicago est dépourvue d'ambiguïté, dans la formulation tardive qu'il en donne dans ses mémoires : "J'arrivais avec l'intention d'apprendre les techniques du métier de sociologue, idées et théories me paraissaient inutiles pour un apprenti sociologue ». Il évoque "le rare privilège de déjeuner avec Burgess", un "survivant de l'école de Chicago ", l'accueil de Wirth qui espérait, écrit-il, qu’il apporterait "des idées», affirme qu'il y "a appris son métier comme [il] le souhaitai $[t]$ et comme on ne pouvait pas le faire à Paris ». Il poursuit : "J'ai eu la chance de participer à une enquête sur l'arrivée des Noirs dans un quartier voisin de l'université : construire le questionnaire et l'échantillon, aller de porte en porte poser les questions ». Il ne mentionne pas le nom de Park, mais celui de Hughes : «ce dernier était alors inconnu en France; au milieu des années 1970, il fut redécouvert par des sociologues de la tradition critique. Sous le nom d'ethnométhodologie, sa démarche est maintenant glorifiée, à mon avis à mauvais escient : c'est trop souvent une justification pour se contenter de descriptions approximatives, une facilité pour ne pas faire de la recherche dure, une pente qui conduit droit à la sociolalie »". Mendras conclut son évocation des séminaires de l'anthroplogue Lloyd Warner (alors à Chicago) et de Hughes ("sur des sujets qui [lui ]paraissaient folkloriques ») : «tout cela ne paraissait pas bien sérieux à l'étudiant parisien qui avait l'habitude de s'asseoir devant une table, sur les bancs de bois de la Sorbonne, pour écouter le maître distiller son savoir " (Mendras 1995: 44, 45, 47-49, 54). Son appréciation est bien différente sur le Bureau of Applied Research de l'Université Columbia « le modèle de tout centre de recherche vivant en marge de l'université grâce à des contrats de recherche ", un modèle dont il affirme qu'il a passé sa vie à essayer de le reproduire.

Un autre sociologue français, Jean-René Tréanton, passa une année à l'Université de Chicago, et s'intéressa à la sociologie du travail de Hughes, longuement citée dans son article du premier numéro de la Revue française de sociologie (Tréanton 1960). Mais Tréanton ne devint pas, contrairement à Mendras, un entrepreneur de recherche important dans les années suivantes et la sociologie du travail de Hughes - aujourd'hui une référence fréquente pour les jeunes sociologues français - ne retint pas l'intérêt jusqu’à la fin des années 1960. L'expérience que firent les autres sociologues français de la sociologie américaine fut évidemment variée, en fonction des lieux de séjours et de leurs propres expériences biographiques et ambitions intellectuelles : par exemple, François Bourricaud et

9. Rappelons qu'il n'y a aucun rapport direct entre Hughes et l'ethnométhodologie qui s'est développée à partir du début des années 1960 autour de Harold Garfinkel, un ancien élève de Parsons en poste à l'Université de Los Angeles. Il est également inexact que Hughes était inconnu en France : il entretint durablement des relations avec Georges Friedmann. 
Alain Touraine séjournèrent à Harvard, le premier traduisit des textes de Parsons, mais le second ne manifesta pas un enthousiasme excessif (Touraine 1977: 64-67) ; Michel Crozier (2002: 105) fut «ébloui » par les psychologues sociaux lors de son séjour à l'Université du Michigan. Les contacts ultérieurs furent surtout fréquents avec le Bureau of Applied Research et les chercheurs de l'Université Columbia. Comme le suggère Henri Mendras, c'est le modèle de la sociologie proposé par le Bureau of Applied Research de Columbia qui a retenu de manière privilégiée l'attention de la majeure partie des jeunes sociologues français. À partir de 1960, l'enquête par questionnaires avec traitement statistique selon le modèle de Lazarsfeld devient la méthode «scientifique » spécifiquement associée à la sociologie. Plusieurs facteurs contribuent à son succès : l'activité inlassable de propagandiste de Lazarsfeld, qui s'appuie sur les crédits de la fondation Ford et a un relais administrativement influent avec Stoetzel ; le développement d'une demande d'enquête des administrateurs modernistes qui gravitent autour du Commissariat au Plan et sont sensibles à la rigueur statistique, qui fournissent les premiers financements de recherche pour la sociologie ${ }^{10}$.

Pour s'établir par rapport aux autres disciplines universitaires et acquérir une légitimité scientifique, les sociologues français sont attentifs à l'appui que peut fournir la statistique : les avantages du modèle de recherche de Columbia sur celui de la tradition de Chicago sont, sous ce rapport, évidents. Quelques sociologues français ont fait du travail de terrain, notamment pour étudier le travail ouvrier, mais aucun de leurs ouvrages n'a acquis une grande notoriété, et cette démarche fut peu valorisée.

Les sociologues de la tradition de Chicago ont été réticents quant à l'utilisation d'enquêtes par questionnaires. Herbert Blumer en est le premier et plus virulent critique, notamment dans son allocution de président de l'Association américaine de sociologie (Blumer 1956). Le principal successeur intellectuel de Park, Everett Hughes, initie à l'époque ses élèves au travail de terrain à la manière des anthropologues, et son enseignement se retrouve dans la grande majorité des monographies d'anthropologie urbaine dont la notoriété croît à la fin des années 1960 (voir Junker 1960 pour le manuel issu de cet enseignement).

On voit que le contexte disciplinaire de la sociologie en France et aux ÉtatsUnis, entre 1945 et 1965, ne favorisait pas l'orientation des jeunes sociologues français vers les apports en termes de démarche qui prolongeaient ceux de la sociologie de Chicago de la période précédente, ce qui n'encourageait pas à un intérêt pour les sujets substantiels traités par ceux-ci.

Les publications, et notamment les traductions de l'anglais - il ne faut pas surestimer les compétences linguistiques des sociologues de ces générations sont, à côté des contacts directs, une médiation éventuelle entre la sociologie américaine et la sociologie française.

10. L'un des signes de ce succès de la définition donnée par Lazarsfeld à la recherche sociologique se trouve dans la référence implicite à ce modèle qui caractérise le manuel de Bourdieu, Passeron \& Chamboredon (1968). 
Les rares traductions d'ouvrages de sociologie publiées avant 1965 confirment ce que suggéraient les emprunts faits par la première génération de sociologues. Elles concernent un recueil d'essais de Parsons (paru en 1955) mentionné plus haut, un autre de Merton (1965) traduit par Mendras, un recueil de textes de psychologie sociale qui connut une diffusion notable, et une série de volumes, versions françaises du manuel de méthodologie de Lazarsfeld et Rosenberg (1957) dont le premier volume au moins connut une certaine diffusion (Boudon \& Lazarsfeld 1965). Une seule traduction en français correspond à un ouvrage qui a un rapport avec la tradition de Chicago : celle du livre du philosophe pragmatiste George Herbert Mead, Mind, Self and Society, parue en 1963. La traduction ne semble pas avoir connu une notable diffusion, de même que l'ouvrage de Franklin Frazier, Bourgeoisie noire, paru en français en 1955 avant une publication en anglais ${ }^{11}$.

Les séparations entre disciplines de sciences sociales ne sont pas, jusqu'en 1965, aussi marquées qu'elles le sont devenues par la suite. Les géographes français n’ont pas ignoré, après 1945, les travaux des sociologues de Chicago, et notamment ceux de Park et d'un de ses élèves, Mackenzie, sur l'écologie des villes (voir Sorre 1957 : 142-156). Les migrations de population à l'intérieur des pays et des continents sont alors pour les géographes un sujet d'étude important, car leur définition de la géographie n'est pas essentiellement nationale et contemporaine (Sorre 1957). Les anthropologues n'ont pas non plus complètement ignoré ces travaux : un article de Claude Lévi-Strauss évoque "les travaux d'écologie urbaine [de l'école dite de Chicago qui] avaient suscité de grands espoirs, trop vite déçus ", sans permettre de conclure que l'auteur en avait une connaissance précise ${ }^{12}$. L'historien de Paris, Louis Chevalier, professeur au Collège de France, mentionne aussi occasionnellement et laudativement les travaux de «l'école de Chicago » (Chevalier 1978 [1958]).

Dans la petite cohorte des premiers sociologues français, l'une des rares références à la tradition de Chicago se trouve dans les travaux de Paul-Henri Chombart de Lauwe (1952) sur Paris, avec une comparaison du schéma de répartition de la population proposé par Burgess à partir de l'exemple de Chicago, et de la répartition spatiale de la population parisienne. Vingt ans plus tard, avec le développement de la sociologie urbaine à la fin des années 1960, la référence aux travaux de Park et Burgess sur les villes devient un peu plus fréquente. Mais il s'agit généralement d'une référence négative et presque routinière à un genre d'analyse qui est considéré comme relevant d'une orientation dépassée, appartenant à l'histoire des approches sociologiques. Les recherches françaises en sociologie urbaine sont alors tournées vers ce qui était un point aveugle dans les analyses de Park et Burgess, comme l'avait d'ailleurs relevé auparavant Franklin Frazier: les relations de pouvoir et la politique urbaine ${ }^{13}$.

11. Quelques articles ont été traduits de l'anglais dans les Cahiers internationaux de sociologie entre 1946 et 1965. Un seul, dans la livraison de 1947, traduction d'un article de l'American Journal of Sociology sur l'écologie humaine, émane d'un sociologue de la tradition de Chicago, Louis Wirth.

12. Lévi-Strauss (1958 : 319). Le texte original avait été publié en anglais en 1953.

13. Voir par exemple Castells (1972); et pour une analyse d'ensemble des orientations de ces recherches, Amiot (1980). 
La traduction d'une partie des essais de The City, en 1979, semble clore cette période de contacts peu fructueux entre la sociologie urbaine et la tradition de Chicago (Grafmeyer \& Joseph 1979).

Cet inventaire des relations entre les sociologues de la tradition de Chicago et les sociologues français de 1945 à 1975 laisse de côté les recherches sur le sujet central de Robert Park. Il montre cependant que les occasions de contacts n'ont pas manqué. Mais dans la phase d'institutionnalisation universitaire où elle se trouvait, la sociologie française se souciait d'acquérir une légitimité scientifique. Pour cela, la tradition de Chicago n'offrait pas de grandes ressources: elle ne proposait pas de modèle prestigieux en matière de démarche de recherche, ni un cadre conceptuel approprié avec sa focalisation sur les villes (et non sur un Étatnation), ni par le public auquel elle s'adressait, les classes moyennes urbaines éclairées (et non les élites de l'administration et du gouvernement).

\section{Les recherches françaises sur les contacts entre populations différentes}

J'examinerai maintenant le petit ensemble de recherches sociologiques françaises qui portent sur un sujet proche des relations de race. Cette expression est absente des Cahiers internationaux de sociologie, de la Revue française de sociologie, et des travaux que je vais évoquer - à l'exception d'un article cité infra. En France, le domaine qui lui correspond à peu près au moins par la définition des populations concernées est celui de l'étude des immigrés et éventuellement celui, emprunté aux géographes, des migrations de population à l'intérieur d'un territoire ${ }^{14}$.

La première étude sociologique sur l'immigration est celle d'une chercheuse du CNRS, Andrée Michel (1956), sur les Algériens en France - un sujet rencontré à l'occasion d'une étude antérieure sur les habitants des hôtels meublés ${ }^{15}$. L'ouvrage décrit, à partir d'une documentation administrative, complétée par une enquête par questionnaires et une autre par entretiens auprès des syndicats et des directions d'entreprise, les conditions de travail et d'habitat des travailleurs émigrés, ainsi que les causes de l'émigration et l'adaptation de ces travailleurs à leur situation (ibid.: 62, 89). Une interrogation centrée sur le travail et le même type de ressources documentaires se retrouve dans un second ouvrage de sociologie publié sur le sujet - issu d'une thèse de troisième cycle (Granotier 1970) ${ }^{16}$.

La Revue française de sociologie a publié un article d'Andrée Michel sur les travaux américains consacrés aux relations de race (Michel 1962). L'auteur s'appuie sur un article critique de Blumer de 1958 qui examine les travaux dans le domaine, dont il reprend, pour une large part, l’appréciation critique.

14. Rappelons que ce sujet est à ce moment à peu près ignoré par les historiens : voir Noiriel (1984). 15. Avant cet ouvrage, la bibliographie sur le sujet se limite à deux thèses de géographie et de droit soutenues avant 1940 : Mauco (1932); Ray (1938).

16. Aucun article sur l'immigration n'est publié dans les Cahiers internationaux de sociologie, mais la revue n'ignore pas l'analyse des situations coloniales, évoquées dans plusieurs articles de Georges Balandier et d'Albert Memmi. On ne trouve dans ces articles que très peu de références aux analyses américaines sur les relations de race. 
Elle évoque quelques études françaises, sans souligner qu’elles sont toutes signées par des géographes ou des anthropologues (Jacques Berque, Georges Balandier, Roger Bastide) et qu'elles portent sur des situations coloniales, et non sur la France métropolitaine. Elle mentionne le recueil des esssais de Park et son " œuvre si novatrice», mais ne remarque pas que la perspective de Park, disparu dix-huit ans auparavant, n'est plus celle qui inspire les travaux sur le sujet qui retiennent alors l'attention aux États-Unis. Elle ne souligne pas la différence dans la définition du domaine : études des relations de races et de culture aux ÉtatsUnis en 1930, étude des émigrés comme travailleurs en France en 1950-1970.

Une thèse un peu plus tardivement publiée, mais dont l'auteur, né en 1926, appartient donc à la génération des jeunes sociologues d'après-guerre, témoigne d'une connaissance précise des travaux des sociologues de Chicago. La Sociologie des migrations aux États-Unis (1974) de René Duchac repose sur des recherches menées aux États-Unis après 1967. L'auteur vise à rendre compte de l'état de la sociologie des migrations inter-urbaines et intra-urbaines et présente les travaux de Burgess et Park, mais aussi ceux de leurs successeurs des années 1950 et 1960, comme les statisticiens Otis D. Duncan, Philip M. Hauser. Hughes et Blumer sont, en revanche, absents de la liste des auteurs cités par Duchac, qui cherche à rassembler en un seul domaine deux des objets principaux de la sociologie de Park - la question des villes et celle de l'hétérogénéité ethnique de la population américaine et de ses conséquences. Son point de vue sur l'immigration reste celui des chercheurs français de la période : alors que Park considère les contacts entre race et culture comme un phénomène d'une grande généralité historique, Duchac les considère comme la conséquence d'une période d'immigration antérieure révolue, relevant du même cadre d'interrogation que les migrations des campagnes vers les villes ou que les mouvements de population entre régions des États-Unis. Ce cadre est exactement celui qu'utilise depuis sa fondation l'INED ${ }^{17}$. Une contribution plus tardive de Duchac à un cahier de l'INED (Duchac 1977) ne garde aucune trace de sa lecture de Park ${ }^{18}$.

Si l'étude de l'immigration n'a que peu de place chez les sociologues du CNRS ou de l'université, elle est au contraire l'un des sujets principaux de l'INED, un institut rattaché au ministère du travail, après 1946, et qui a hérité en partie de la fondation Carrel créée sous Vichy. Ces travaux ont pour prédécesseur la thèse de géographie du sulfureux Georges Mauco, spécialiste des étrangers en France, passé du Parti populaire français de Jacques Doriot au Haut comité de la population française de la Libération, dont l'INED publia les contributions.

17. On trouve chez René Duchac des remarques surprenantes, par exemple: «dans notre pays, l'immigrant est beaucoup plus reçu qu'appelé. S'il vient en France [...] ce n'est jamais à la faveur d'une propagande suscitée par les instances politiques du pays [...] et qui l'inviterait à venir peupler le "désert français"..." (1974: 27).

18. Signalons aussi l'ouvrage de Charlotte Roland sur deux générations de juifs installés à Belleville (1962), dont le préfacier Louis Chevalier souligne la similitude d'inspiration avec les travaux américains sur les groupes ethniques de Chicago. Relevant de l'histoire démographique à la manière de son préfacier, publié dans une collection de livres sur le judaïsme, il semble n’avoir eu qu'une audience réduite et peu durable. 
La question de l'immigration est présente dès la fondation de l'INED : elle fait l'objet d'un article du directeur de la revue, Alfred Sauvy, dans la première livraison de Population. Il y affirme la nécessité pour la France d'une immigration importante, et souligne qu'il ne s'agit pas seulement d'une émigration temporaire de travailleurs :

«L'admission sur notre sol de nouveaux étrangers ne doit pas être considérée [...] comme une simple admission temporaire de travailleurs [...] mais comme une véritable immigration, de personnes appelées à rester et à faire souche " (1946: 97).

L'article qui suit dans le même numéro de Population introduit un nouveau thème, promis à un long avenir : les facteurs comparés d'assimilation pour deux populations (les Russes et les Arméniens). Dans les années suivantes, en 1947, 1953 et $1954^{19}$, les recherches sur l'immigration occupent trois volumes des cahiers de l'INED . À l'arrière-plan de ces recherches se trouve toujours la question de la nécessité d'un apport de main-d'œuvre pour l'économie française. Les recherches portent sur les «attitudes » de la population autochtone et des populations immigrées l'une par rapport à l'autre (pour les recherches d'Alain Girard et de Jean Stoetzel), et sur la question de l'assimilation des immigrés à la population française. L'avancée de cette assimilation est scrutée et considérée comme inéluctable, avec cependant une incertitude dans le cas des émigrations du Maghreb. La désignation de ces populations est hésitante: parfois des termes géographiques - "Nord-africains » et "Algériens ", puisque les Algériens sont alors citoyens français et non soumis aux lois sur l'émigration si bien d'ailleurs que leurs déplacements d'un bord à l'autre de la Méditerranée ne sont pas bien connus -; parfois des termes ethniques - Arabes, Berbères, Chleuh, dans un article qui estime leurs mérites différentiels en tant que travailleurs (Sanson 1947 : 177-178) ; parfois encore une qualification religieuse (musulmans). Une autre hésitation significative de la terminologie, relevée par certains chercheurs, porte sur l'«assimilation ", l'" adaptation ", l'« intégration ", etc., avec des qualificatifs comme "politique», "sociale». La notion centrale de Park, relations de race, n'est jamais utilisée.

Ces difficultés d'élaboration d'une perspective de sciences sociales pour analyser l'arrivée sur le territoire français de nouvelles populations et leur avenir sont révélatrices des représentations de base de la réalité sociale que les chercheurs français partagent avec les dirigeants politiques, et sans doute avec une grande partie des catégories intellectuelles de l'époque. Celles-ci apparaissent par exemple derrière cette remarque de Louis Chevalier, dans un texte, daté de janvier 1944, qui sert d'introduction au deuxième cahier de l'INED (Chevalier 1947 : 11-14), et se donne comme un préalable à une politique d'immigration de travailleurs :

19. Cf. : Louis Chevalier et al. (1947) ; Girard \& Stoetzel (1953 et 1954). Voir aussi deux courts articles: Poignant (1949) et Lecarpentier (1949). Une bibliographie exhaustive des travaux de l'INED sur l'immigration se trouve dans le Cahier 79 de l'INED (Institut national d'études démographiques 1977). 
" [...] ces étrangers ne doivent pas, par leur entrée massive en France et leur installation sur notre sol, risquer de changer les valeurs physiques, spirituelles et morales auxquelles nous tenons [...] ces étrangers ne sont pas destinés seulement à faire des travailleurs, mais encore, par une assimilation qu'il restera à définir dans ses moyens, à faire des Français et à accroître les chances d'une continuation spirituelle et morale et d'un renouvellement spirituel et moral du pays».

L'expérience sociale antérieure de l'émigration en France, que n’ignorent pas les chercheurs de l'INED - l'existence d'une émigration importante, principalement belge et allemande depuis le début du XIX ${ }^{\mathrm{e}}$ siècle, qui s'est rapidement fondue dans la population française - fournissait évidemment une justification à la perception de cet avenir.

Cette représentation de base repose sur la reconnaissance implicite d'un cadre national (relativement) immuable dans sa définition "morale " - en un mot conforme au modèle de la République selon les principes de 1789, c'est-à-dire une organisation de l'État sur un territoire avec une population homogène ou destinée à le devenir rapidement - et sur un mythe historique, celui de la constitution nationale du territoire et de la nation. On doit souligner que le cadre d'interrogation des sciences sociales, celui des démographes de l'INED, comme celui des sociologues au moins jusque dans les années 1970, est un cadre national. Tous se donnent pour objet de contribuer à une sociologie de la France. Dans le contexte des années 1945-1960, où par ailleurs la conception biologique des différences entre populations ne peut être avancée publiquement qu'avec prudence, notamment par les héritiers de la fondation Carrel, on comprend qu'il y ait eu place pour des études sur les émigrés comme population et sur leur avenir inéluctable comme assimilés, mais que les relations de race ou de groupes ethniques comme phénomène mondial récurrent soient occultées. Cette orientation laissait pourtant de côté des phénomènes alors visibles : les conséquences concrètes du passé colonial, ce qui va devenir les Départements d'Outre-mer, et la question de l'Algérie. On peut d'ailleurs remarquer que les chercheurs de l'INED ont hésité sur la possibilité d'une assimilation des travailleurs d'origine africaine ${ }^{20}$ : l'interrogation sur l'assimilation des Polonais et des Italiens est moins inquiète, et l'on peut trouver à l'occasion l'expression d'une nostalgie pour les périodes où l'on pouvait recruter des travailleurs nouveaux chez «nos fournisseurs» habituels nordiques, notamment les Belges et les Hollandais (Lecarpentier 1949).

Jusqu'en 1980 au moins, la question de l'immigration est ainsi considérée par la sociologie française comme d'intérêt sectoriel : dans les deux ouvrages issus de colloques réunissant sociologues et économistes sur les évolutions de la société

20. Voir par exemple Ray (1949), ainsi que les formulations prudentes du Cahier 20 de l'INED (Girard \& Stoetzel 1954) à propos de l'immigration familiale: "On ne recherche ici ni ses causes ni ses composantes. On la considère comme un fait et l'on essaie de saisir si une adaptation des familles musulmanes aux modes de vie français se fait ou non, dans quelle mesure et comment " (p. 97); «Si l'on considère l'adaptation comme un processus qui ne transforme pas un immigrant en un autochtone [...], il semble que toutes solutions aux difficultés présentes, et aux tensions qui peuvent en résulter ne soit pas exclue» (p. 144). 
française dans les années 1960 (Darras 1966 ; Reynaud 1966), la question n’apparaît à peu près pas; elle n'apparaît toujours pas, treize ans plus tard, dans le colloque qui prolonge l'un des précédents (Mendras, ed. 1980) ${ }^{21}$. L'immigration est considérée comme une question marginale, qui concerne essentiellement le travail, et l'avenir attribué aux groupes d'immigrés est essentiellement une dilution sans laisser de trace, l'assimilation, même si l'on s'interroge à l'occasion sur l'«assimilabilité" de telle immigration ${ }^{22}$. Cette conception s'oppose à celle des chercheurs américains de la génération de Park, comme des suivantes, où l'existence de populations qui s'estiment et sont considérées comme différentes les unes des autres apparait durable, sinon définitive, et où les conflits de races ont un caractère immédiatement visible dans la sphère publique.

\section{Deux conceptions divergentes de la structure sociale}

On a vu les tentatives de Halbwachs pour réinterpréter en termes de différences de classes, les différences ethniques mises en avant par les recherches conduites autour de Park et Burgess. C'est probablement ce qu'aurait fait la plus grande partie des spécialistes en sciences sociales français, entre 1930 et 1970. Ceux-ci partagent la conviction qu'il existe comme éléments relativement stables dans le temps un ensemble de groupes sociaux distincts, définis par leur position économique et qui sont associés à des intérêts et à des comportements spécifiques dans différents domaines. D'où l'introduction, dans toute enquête sociologique, d'une interrogation sur les conséquences potentielles des différences de classe. Il convient ici de ne pas attacher trop d'importance aux interminables débats sur la notion de classe alimentés par le personnel politique, le mouvement ouvrier et plus tardivement par les spécialistes en sciences sociales (voir Portis 1988 pour une vue d'ensemble). Les débats qui ont agité la sociologie - notamment dans les années 19501970 -, ainsi que les références négativement ou positivement enflammées au marxisme, ont en partie oblitéré ce qu'il y avait de commun dans la conception de la structure sociale à laquelle se réferent les sociologues français ${ }^{23}$. Même s'il existe

21. Voir l'analyse convergente de Pierre-Jean Simon (1982-1983) qui porte aussi sur le cas de l'anthropologie.

22. C'est aussi ce que soutient Louis Chevalier dans sa préface de l'ouvrage de Charlotte Roland (voir supra) : «Il n'y a jamais eu d'étude de l'intégration des groupes étrangers à la société française et plus spécialement au milieu parisien, parce que cette intégration n'a jamais posé de grands problèmes, et surtout parce que l'opinion n'y a jamais prêté attention : l'opinion la plus commune, celle de la rue, de l'atelier, de l'usine, mais aussi l'opinion la plus haute qui se reconnaît en général à ce qu'elle ne differe guère de la première, sa principale qualité étant la mise en forme de ce que le bon peuple pense » (Roland $1962: 11$ ).

23. Les numéros de 1965 des Cahiers internationaux de sociologie sont entièrement occupés par un débat sur le sujet avec des contributions de Raymond Aron, Henri Lefebvre, S. Mallet, etc. Voir aussi les cours de sociologie professés à la Sorbonne dans les années 1960 (publiés postérieurement) de Raymond Aron (1964) et Georges Gurvitch (1971) ; et, pour l'avant-guerre, Raymond Aron et al. (1939). 
des désaccords sur les principes des distinctions, les observateurs s'accordent à peu près sur les grands groupes qu'il convient de distinguer, que l'on trouve déjà d'ailleurs dans Les Luttes de classes en France de Karl Marx pour la France de 1850 : une vaste classe ouvrière, en face d'une bourgeoisie possédante, d'une paysannerie nombreuse, et de classes moyennes (au singulier ou au pluriel) ${ }^{24}$. Pour ceux qui font des recherches empiriques, cette convergence des catégorisations tient aussi à l'existence du code des catégories socioprofessionnelles (CSP) de l'INSEE, et, plus profondément à la nécessité pratique de désigner des sortes d'agrégats de populations aux contours un peu flous, au moins pour certaines périodes, à partir de caractéristiques relativement visibles et donc pour partie institutionnalisées : la dimension économique occupe inévitablement une place importante dans cette caractérisation - et plutôt le métier que le patrimoine, moins accessible le plus souvent pour l'enquêteur (Chapoulie 1985). On peut relever que la notion de genre est absente des définitions de la structure sociale, tout comme celle de génération; celles-ci sont sans doute considérées comme des caractéristiques transversales à la structure sociale et secondaires.

Une sorte d'écho de cette représentation se trouve dans le premier découpage des domaines à conquérir par la sociologie comme nouvelle discipline universitaire. Henri Mendras, dans ses mémoires, évoque ce découpage à propos du choix de son sujet de recherche: "Surtout, comme toute ma génération, j'étais [...] préoccupé par la question ouvrière, vers laquelle Friedmann nous avait tous orientés. Mais celui-ci me dit: "Mendras, Touraine c'est les ouvriers, Tréanton la ville, Crozier les fonctionnaires. Vous, vous comprenez quelque chose aux paysans et vous êtes le seul, alors, occupez-vous en” " (1995: 53). Ce découpage en domaines de recherches se distingue de celui que révèlent les thèses soutenues à Chicago dans les années 1923-1930 (la liste se trouve dans Faris 1970). Une seule thèse, consacrée au Japon dont son auteur était originaire, porte dans son titre le terme «classe sociale »; en revanche, de nombreuses thèses ont pour objet les groupes ethniques: l'une sur l'assimilation des émigrés tchèques, une autre sur les émigrés suédois, une autre sur le ghetto juif, une thèse porte sur les mulâtres, enfin plusieurs sur les préjugés de race et sur les Noirs.

La sociologie américaine entre 1920 et 1940 (et au-delà) est en effet toujours très attentive à la différenciation ethnique, mais elle ignore presque complètement les différences de classe. Dans la représentation de la société américaine, la différenciation principale correspond à l'existence de groupes définie par une origine (supposée) commune. Les emplois occupés par les membres de ces groupes dont l'existence est jugée durable ne sont pas des caractéristiques considérées comme pérennes, et un processus général de mobilité ascendante dans

24. Aron (1964: 80-82) remarque que la structure de classe selon l'analyse proposée par Lloyd Warner pour les villes américaines s'applique assez bien à la ville du Havre telle qu'il l'avait connue, c'est-à-dire - mais il n'attire pas lui-même là-dessus l'attention - selon la perception d'un professeur de philosophie de lycée qui s’appuie exclusivement sur des observations personnelles sommaires. 
l'emploi concerne - ou plutôt est supposé concerner - une partie significative de leurs membres dans une société où les évolutions de l'emploi sont rapides. Par ailleurs, l'histoire du mouvement ouvrier, avec une opposition durable entre travailleurs qualifiés anglo-saxons et nordiques réunis dans des syndicats de métiers, et ouvriers non qualifiés d'autres origines ethniques, longtemps dépourvus de représentation syndicale, ne favorise pas une perception de la structure sociale dont la classe ouvrière serait l'un des éléments principaux.

Robert Park ignore presque complètement la notion de classe sociale, dont il affirme à l'occasion qu'elle ne peut s'appliquer pour les États-Unis, même si on la trouve dans la surprenante phrase finale d'un de ses principaux essais sur les relations de race en $1939^{25}$. Park utilise une sorte de substitut à la notion de classe dans des analyses imprécises en terme de «statut ». Comme je l'ai montré ailleurs (Chapoulie 2000), cette omission procède de plusieurs éléments. Park était politiquement conservateur, et on trouve sous sa plume de nombreuses remarques qui suggèrent une adhésion au "rêve américain " de mobilité sociale - même si c'est l'héritage de son père qui lui permit de devenir enseignant en sociologie presque sans rémunération en 1913. Deuxièmement, le contexte politique des années 1919-1930 est particulièrement défavorable au mouvement ouvrier américain, très affaibli à partir de la fin de la guerre, avec une période de prospérité économique exceptionnelle et un syndicalisme pourchassé jusqu'au New Deal. Évoquer le terme "classe sociale " était à peu près exclu pour un sociologue américain des années 1920, et insister sur le fondement de celle-ci dans la place de la division du travail certainement peu susceptible de valoir un quelconque crédit à un chercheur (Gilkeson 1995) ${ }^{26}$. La notion de classe sociale, utilisée avant 1914 par le fondateur du département de sociologie de Chicago, Albion Small, retrouve une place dans la sociologie américaine après 1935, avec les études de communautés américaines par l'anthropologue Lloyd Warner qui définit la notion à travers le système de relations sociales. On la trouve également chez Franklin Frazier et Everett Hughes (voir Hughes \& Hughes-MacGill 1952). Cette réintroduction prudente de la notion de classe sociale en sociologie accompagne une transformation des représentations de la société américaine associée à la crise, mais aussi à l'arrêt de l'immigration et à l'importance croissante du "problème noir ". Dans ces conditions nouvelles, la mobilité collective des groupes d'immigrés est moins frappante qu'elle ne l'était précédemment. À l'explication antérieure de la délinquance comme associée à une étape dans l'installation aux États-Unis (typique des élèves de Park et Burgess), se substitue également une explication par la position sociale. Cependant, même après 1940, il existe encore des obstacles qui vont, dans les sciences sociales, à l'encontre d'un accent sur la structure de classe : un nouvel obstacle sociopolitique dans les années du maccarthysme, mais

25. Cet essai s'achève par la phrase «Les conflits de race dans le monde moderne seront, dans le futur, confondus et en fin de compte supplantés par les conflits de classe» (Park 1950 : 116).

26. L'une des rares exceptions d'usage de la notion de classe avant la crise est l'ouvrage des Lynd (1929). 
aussi la grande diversité des villes à l'intérieur d'un vaste territoire comme celui des États-Unis qui rend difficilement comparable leurs populations.

Les traductions en nombre croissant à partir de 1980, ainsi que les références devenues fréquentes à des publications qui se rattachent à la tradition de Chicago, témoignent d'une diffusion tardive qui s'étend un peu au-delà des sociologues. Celle-ci semble être passée par deux canaux principaux. Le premier est l'intérêt, et parfois l'usage, des autobiographies en sociologie après 1968. Un rapport de recherche de Daniel Bertaux (1996), non publié, semble avoir été un intermédiaire. Un second canal est celui des travaux ethnographiques sur le travail, la médecine, la délinquance de la troisième génération de chercheurs de la tradition de Chicago. On ne peut être surpris que les analyses de ces derniers représentants de la tradition de Chicago, qui portaient sur des sujets d'actualité en France, aient connu une diffusion en France plus rapide que leurs devanciers, ni d'ailleurs de ce que les démarches de recherche - recueil de biographies, observation - aient constitué des étapes avant l'appropriation de certaines des analyses substantielles. Quant à la sociologie des relations de race de Robert Park, la partie la plus élaborée des analyses de celui-ci, elle est restée à l'écart de toute diffusion (et il existe après 1980, quand le sujet devient d'actualité en France, bien d'autres sources d'emprunts possibles dans les travaux récents). À demi oubliée aux États-Unis après 1965, elle a laissé la place à des analyses dont les éléments de base ne sont pas toujours très différents de ceux proposés par Park, mais qui sont associés aux interrogations publiques actuelles sur un sujet redevenu d'actualité.

L'emprunt tardif des sources documentaires dont la tradition de Chicago illustrait les usages montre qu'un filtre dans l'emprunt entre traditions nationales différentes réside dans la conjoncture intellectuelle et politique dans laquelle se trouve la discipline emprunteuse. Sa non-diffusion dans la période antérieure à 1980 ne s'explique pas, on l'a montré, par une simple ignorance: il y eut des contacts entre chercheurs qui pouvaient servir de canaux et des chercheurs pour lire attentivement les analyses de Park. L’obstacle a résidé en partie dans les différences de définition des problèmes publics auxquels sont confrontés les sociologues des deux pays. Mais aussi, comme on vient de le voir, dans les différences entre les représentations de la structure sociale sur laquelle repose l'approche de Park et celles, en termes de classes sociales, qui ont inspiré durablement la sociologie française.

Soulignons pour terminer qu'on a utilisé, sans le mentionner explicitement, un troisième terme de comparaison : une comparaison entre la sociologie française de 1930-1980 et l'état actuel de la sociologie française, qui accorde dorénavant une place conséquente aux études sur la présence de populations diverses sur le territoire national. Ce n'est en effet que dans une démarche comparative que l'on peut trouver des ressources pour objectiver des propriétés qu'un examen isolé, si approfondi soit-il, des cadres d'analyse d'une discipline dans une période 
donnée, ne peut révéler. Et ainsi apporter une contribution à l'analyse des catégories de base des sciences sociales, en précisant ce qui en fait des "semi-noms propres » pour emprunter la terminologie de Passeron (2006 [1991] : 130), c'està-dire comportant "une référence tacite à des coordonnées spatio-temporelles ".

Université Paris 1 - Panthéon-Sorbonne Groupe de recherche École, Travail, Institution, Paris jean-michel.chapoulie@univ-paris1.fr

MOTS CLÉS/KEYWORDS : histoire des sciences sociales/history of social sciences - conceptualisation - école de Chicago/Chicago school - relations interethniques/ethnic relations - relations de race/race relations - Robert Park.

BIBLIOGRAPHIE

Amiot, Michel

1980 Contre l'État, les sociologues. Paris, Éd. de l'Ehess.

Aron, Raymond

Blumer, Herbert

1955 «Reflections on Theory of Race Relations ", in Andrew Lind, ed., Race Relations in World Perspective. Honolulu, University of Hawaii Press : 3-21.

1964 La Lutte des classes. Paris, Gallimard.

Aron, Raymond et al.

1956 "Sociological Analysis and the

"Variable" ", American Sociological Review 22 (6) : 683-690.

1939 Inventaire, 3. Les classes moyennes. Paris, Alcan.

1958 "Research on Racial Relations, United States of America ", International Social Science Bulletin 10 (3) : 403-447.

Balandier, Georges

1954 "Sociologie de la colonisation et relations entre sociétés globales ", Cahiers internationaux de sociologie 17 : 17-31.

\section{Bertaux, Daniel}

1996 Histoire de vie ou récits de pratiques? Méthodologie de l'approche biographique en sociologie. Rapport multigraphié pour le CORDES, Paris. [Inédit.]

Bloch, Marc

1992 [1949] Apologie pour l'histoire. Paris, Armand Colin.

Boudon, Raymond \& Paul Lazarsfeld, eds

1965 Le Vocabulaire des sciences sociales: concepts et indices. Paris-La Haye, Mouton.

Bourdieu, Pierre

1997 Méditations pascaliennes. Paris, Le Seuil («Liber »).

Bourdieu, Pierre, Jean-Claude Passeron \& Jean-Claude Chamboredon

1968 Le Métier de sociologue. Paris-La Haye, Mouton. 
Castells, Manuel

1972 La Question urbaine. Paris, Maspero.

\section{Cavan, Ruth Shonle}

1928 Suicide. Chicago, University of

Chicago Press.

\section{Chapoulie, Jean-Michel}

1985 «Remarques sur les relations entre analyses en termes de rapports de classe et études empiriques de catégories sociales par enquêtes statistiques", in Classes et catégories sociales. Aspects de la recherche. Colloque (Paris, 4-5 décembre 1984). Roubaix, Edires : 107-117.

1991 «La seconde fondation de la sociologie française, les États-Unis et la classe ouvrière ", Revue française de sociologie 32 (3) : 321-364.

1999 «Robert E. Park, la tradition de Chicago et l'étude des relations entre les races ", Sociétés contemporaines 33-34: 139-157.

2000 "L'étrange carrière de la notion de classe sociale dans la tradition de Chicago en sociologie ", Archives européennes de sociologie 46 (1) : 53-70.

2001 La Tradition sociologique de Chicago, 1892-1961. Paris, Le Seuil.

2005 «Un cadre d'analyse pour l'histoire des sciences sociales ", Revue d'histoire des sciences humaines 13: 99-126.

2008 «Une interprétation de la sociologie de Robert E. Park dans son contexte historique ", in Suzie Guth, ed., Modernité de Robert Park. Paris, L'Harmattan : 133-154.

\section{Chevalier, Louis}

1947 «Principaux problèmes de l'immigration ", in Louis Chevalier et al., Documents sur l'immigration. Paris, PUF ( Travaux et Documents. INED ; Cahier $2 »)$ : 11-23.

1978 [1958] Classes laborieuses et classes dangereuses à Paris, pendant la première moitié du XIX siècle. Paris, Le Livre de Poche.
Chevalier, Louis et al.

1947 Documents sur l'immigration. Paris, PUF ( Travaux et Documents. INED;

Cahier $2 »)$.

Chombart de Lauwe, Paul-Henri, ed.

1952 Paris et l'agglomération parisienne. Paris, PUF : I, 40-53.

Crozier, Michel

2002 Mémoires. Paris, Fayard.

Darras (groupe d'Arras)

1966 Le Partage des bénéfices. Paris, Minuit.

Duchac, René

1974 La Sociologie des migrations aux ÉtatsUnis. Paris-La Haye, Mouton.

1977 « Facteurs urbains de l'adaptation des immigrés maghrébins ", in Les Immigrés du Maghreb. Études sur l'adaptation en milieu urbain. Paris, PUF ("Travaux et documents. INED ; 79») : 5-132.

Durkheim, Émile

1975 Textes, 3. Fonctions sociales et institutions. Paris, Minuit.

\section{Faris, Robert E.}

1970 Chicago Sociology, 1920-1932. Chicago, University of Chicago Press.

Frazier, Franklin

1949 «Race Contacts and the Social Structure ", American Sociological Review $14(1): 1-11$.

1955 Bourgeoisie noire. Paris, Plon.

\section{Gilkeson, John S.}

1995 "American Social Scientists and the Domestication of "Class", 1929-1955", Journal of the History of the Behavorial Sciences $31: 331-346$.

Girard, Alain \& Jean Stoetzel, eds 1953 Français et Immigrés, 1. L'attitude française, l'adaptation des Italiens et des Polonais. Paris, PUF («Travaux et documents. INED ; $19 »)$ 
1954 Français et Immigrés, 2. Nouveaux documents sur l'adaptation, Algériens, Italiens,
Paris, PUF ( Travaux et documents. INED ; $20 »)$.

Grafmeyer,Yves \& Isaac Joseph, eds 1979 L'École de Chicago. Naissance de l'écologie urbaine. Paris, Champ urbain.

\section{Granotier, Bernard}

1970 Les Travailleurs immigrés en France. Paris, Maspéro.

\section{Gurvitch, Georges}

1971 [1966] Études sur les classes sociales. Lidée de classe sociale de Marx à nos jours. Paris, Gonthier.

Halbwachs, Maurice

1932 "Chicago, expérience ethnique", Annales d'histoire économique et sociale 4 (3) : 11-49.

\section{Hughes, Everett $\mathrm{C}$.}

1943 French Canada in Transition. Chicago, University of Chicago Press /Toronto, W. J. Cage \& $\mathrm{C}^{\mathrm{ie}}$. [Trad. franç. par Jean-Charles Falardeau : Rencontre de deux mondes. La crise d'industrialisation du Canada français, Montréal, Parizeau, 1945.]

1963 « Race Relations and the Sociological Imagination ", American Sociological Review 28 (6) : 897-890.

Hughes, Everett C. \& Helen Hughes-MacGill 1952 Where Peoples Meet. Racial and Ethnic Frontiers. Glencoe, The Free Press.

Institut national d'études démographiques 1977 Les Immigrés du Maghreb. Études sur l'adaptation en milieu urbain. Paris, PUF («Travaux et documents. INED; 79»).

Junker, Buford $\mathrm{H}$.

1960 Field Work: An Introduction to the Social Sciences. Chicago, University of Chicago Press.
Lazarsfeld, Paul \& Morris Rosenberg, eds

1957 The Language of Social Research.

Glencoe, The Free Press.

Lecarpentier, G.

1949 "Les agriculteurs hollandais dans

l'Yonne", Population 3 (1) : 163-168.

\section{Lévi-Strauss, Claude}

1958 Anthropologie structurale. Paris, Plon.

Lynd, Robert S. \& Helen Merrell Lynd

1929 Middletown. A Study in Modern American Culture. New York, Harcourt Brace.

\section{Mauco, Georges}

1932 Les Étrangers en France: leur rôle dans l'activité économique. Paris, Armand Colin.

\section{Mead, George Herbert}

1963 L'Esprit, le soi et la société. Paris, PUF. [Trad. franç. de Mind, Self, and Society, Chicago, University of Chicago Press, 1934.]

\section{Mendras, Henri}

1995 Comment devenir sociologue. Souvenirs d'un vieux mandarin. Paris, Actes Sud.

Mendras, Henri, ed.

1980 La Sagesse et le Désordre. Paris, Gallimard.

Michel, Andrée

1956 Les Travailleurs algériens en France. Paris, Éd. du CNRS.

1962 "Tendances nouvelles de la sociologie des relations raciales ", Revue française de sociologie 3 (2) : 181-190.

Noiriel, Gérard

1984 Le Creuset français. Paris, Le Seuil.

Park, Robert

1950 [1939] "The Nature of Race Relations ", in The Collected Papers of Robert Ezra Park, 1. Race and Culture. Edited by Everett Hughes et al. Glencoe, The Free Press : 81-116. 
1950 Collected Papers of Robert Ezra Park, 1. Race and Culture. Edited by Everett Hughes et al. Glencoe, The Free Press.

1952 Collected Papers of Robert Ezra Park, 2. Human Communities: The City and Human Ecology. Edited by Everett Hughes et al. Glencoe, The Free Press.

1955 Collected Papers of Robert Ezra Park, 3. Society: Collective Behaviour, News and Opinion and Modern Society. Edited by Everett Hughes et al. Glencoe, The Free Press.

\section{Park, Robert E. \& Ernest W. Burgess}

1921 Introduction to the Science of Sociology. Chicago, University of Chicago Press.

1925 The City. Chicago, University of Chicago Press.

Passeron, Jean-Claude

2006 [1991] Le Raisonnement sociologique. Paris, Albin Michel.

\section{Poignant, Raymond}

1949 «Étude sur l'assimilation de l'immigration polonaise dans le Pas-de-Calais ", Population 3 (1) : 157-162.

\section{Portis, Larry}

1988 Les Classes sociales en France, un débat inachevé, 1789-1989. Paris, Éditions ouvrières.

\section{Ray, Joanny}

1938 Les Marocains en France. Paris, Sirey.

1949 «Les Nord-Africains en Meurthe-et Moselle", Population 3 (2) : 368-370.

Reynaud, Jean-Daniel, ed.

1966 Tendances et volontés de la société française. Paris, SEDEIS.

\section{Roland, Charlotte}

1962 Du ghetto à l'Occident, deux générations yiddiches en France. Préface de Louis Chevalier. Paris, Minuit.

\section{Sauvy, Alfred}

1946 «Évaluations des besoins de l'immigration française", Population 1 (1) : 91-99.

\section{Sanson, Robert}

1947 «Les travailleurs nord-africains de la région parisienne", in Louis Chevalier et al., Documents sur l'immigration. Paris, PUF

(«Travaux et Documents. INED ;

Cahier $2 »):$ 162-193.

\section{Simon, Pierre-Jean}

1982-1983 «L'étude des problèmes des minorités et des relations inter-ethniques dans l'anthropologie et la sociologie françaises ", Pluriel 32-33 : 13-25.

\section{Sorre, Maximilien}

1955 Les Migrations des peuples. Essai sur la mobilité géographique. Paris, Flammarion.

1957 Rencontre de la géographie et de la sociologie. Paris, Marcel Rivière.

\section{Topalov, Christian}

2005a «Un savant voyage : les "Lettres des États-Unis" de Maurice Halbwachs au Progrès de Lyon (septembre-décembre 1930) ", Genèses 58 (1) : 132-150.

2005 b «Un savant voyage : les "Lettres des États-Unis" de Maurice Halbwachs au Progrès de Lyon (septembre-décembre 1930)", Genèse 59 (2) : 131-150.

2006a «Maurice Halbwachs. L'expérience de Chicago (automne 1930)", Annales 61 (3) : 555-581.

2006b «Maurice Halbwachs et les sociologues de Chicago ", Revue française de sociologie 47 (3) : 561-590.

\section{Touraine, Alain}

1977 Un désir d'histoire. Paris, Stock.

Tréanton, Jean-René

1960 "Le concept de carrière ", Revue française de sociologie 1 (1) : 73-80. 
Jean-Michel Chapoulie, Malentendus transatlantiques: la tradition de Chicago, Park et la sociologie française. - Que peut apprendre sur les conceptions de base de la sociologie française la non-réception des analyses de Robert Park sur les relations de race et de culture qui constitue l'élément central et le plus élaboré de la tradition de Chicago en sociologie? L'article analyse les contacts de la sociologie française de Maurice Halbwachs jusqu'à la première génération des sociologues français de l'Après-guerre, ainsi que les travaux français sur l'immigration de 1945 à 1970. Il montre que l'incompréhension entre chercheurs français et américains repose sur deux conceptions opposées de la structure sociale qui sont le produit de leurs expériences historiques nationales respectives: en termes de classes sociales chez les premiers, de groupes ethniques chez les seconds. Un second élément favorable à cette non-réception est également mis en évidence : le contexte du développement de la sociologie française jusqu'en 1970 et sa quête d'une légitimité scientifique.
Jean-Michel Chapoulie, Transatlantic Misunderstandings: The Chicago School, Robert Park and French sociology. — What can we learn about French sociology's fundamental conceptions by inquiring into the reasons why it failed to receive Robert Park's analyses of race relations and culture, a key element (and the one most worked on) in the Chicago school of sociology? This inquiry into the transatlantic contacts of French sociology from Maurice Halbwachs till the first generation of sociologists following WW II focuses on French studies of immigration from 1945 to 1970 . The misunderstanding between French and American social scientists was underpinned by opposite conceptions of "social structure", stemming from their respective national experiences: social classes for the former, and ethnic groups for the latter. Other factors accounting for the aforementioned lack of reception were : the context in which French sociology developed till the 1970 s and its quest for scientific legitimacy. 